\title{
Finite-temperature three-point function in 2D CFT
}

\section{Melanie Becker, Yaniel Cabrera and Ning Su}

George and Cynthia Mitchell Institute for Fundamental Physics and Astronomy, Texas A $83 M$ University, College Station, TX 77843-4242, U.S.A.

E-mail: mbecker@physics.tamu.edu, cabrera@physics.tamu.edu, suning1985@gmail.com

ABSTRACT: We calculate the finite temperature three-point correlation function for primary fields in a 2D conformal field theory in momentum space. This result has applications to any strongly coupled field theory with a 2D CFT dual, as well as to Kerr/CFT.

KEYworDS: Gauge-gravity correspondence, AdS-CFT Correspondence, Conformal Field Models in String Theory

ARXiv EPRINT: 1407.3415 
With the discovery of AdS/CFT [1] and generalizations thereof like AdS/CMT [2-5], Kerr/CFT [6-8] and the applications of holographic duality to QCD [9], the calculation of finite-temperature correlation functions in conformal field theory has become increasingly important.

A lot of these holographic dualities contain black holes and thus describe dual field theories at finite temperature. For practical applications it is often convenient to work in momentum space. For example, even before the era of AdS/CFT it was noticed by Maldacena and Strominger [10] that the semiclassical emission rates of scalar fields in a Kerr/Newmann black hole agree with the result obtained from a 2D effective conformal field theory in momentum space. The generalization to photons and fermions was later worked out by Gubser [11].

In many cases the theory of interest is strongly coupled, so the conformal field theory calculation is the only reliable method to compute correlation functions. This is the point of view we take in this short note.

We are interested in finite temperature three-point functions for primary fields in 2D CFT in momentum space. First, we recall the calculation of the two-point function (see [1] and references therein). At zero temperature, the coordinate representation of this correlator for two primary fields $\phi_{h_{i}, \bar{h}_{i}}$ with conformal weights $\left(h_{i}, \bar{h}_{i}\right), i=1,2$, has the familiar form on the complex plane (after normalization)

$$
\left\langle\phi_{h_{1}, \bar{h}_{1}}(z, \bar{z}) \phi_{h_{2}, \bar{h}_{2}}(0,0)\right\rangle=\frac{\delta_{h_{1}, h_{2}} \delta_{\bar{h}_{1}, \bar{h}_{2}}}{z^{2 h_{1}} \bar{z}^{\overline{2} h_{1}}} .
$$

We compactify this result on cylinders with radii $T_{L}$ and $T_{R}$ via the mapping

$$
z=e^{2 \pi T_{L} w}, \quad \bar{z}=e^{2 \pi T_{R} \bar{w}},
$$

where $T_{L}, T_{R}$, are the left and right moving temperatures. The imaginary part of $w=x+i \tau$ is the compactified time direction such that $\tau \sim \tau+1 / T_{L}$. This mapping gives the finitetemperature two-point function in coordinate space

$$
\left\langle\phi_{h_{1}, \bar{h}_{1}}(\omega, \bar{\omega}) \phi_{h_{2}, \bar{h}_{2}}(0,0)\right\rangle=\left(\frac{\pi T_{L}}{\sinh \pi T_{L} w}\right)^{2 h_{1}}\left(\frac{\pi T_{R}}{\sinh \pi T_{R} \bar{w}}\right)^{2 \bar{h}_{1}},
$$

where we used $h_{1}=h_{2}$ and $\bar{h}_{1}=\bar{h}_{2}$; otherwise the correlator vanishes.

To compute the propagators we Wick rotate $\tau \rightarrow i t$ to obtain the light-cone coordinates $x^{ \pm}=x \pm t$. We obtain the Lorentzian two-point function

$$
\left\langle\phi_{h_{1}, \bar{h}_{1}}\left(x^{+}, x^{-}\right) \phi_{h_{2}, \bar{h}_{2}}(0,0)\right\rangle=\left(\frac{\pi T_{L}}{\sinh \pi T_{L} x^{-}}\right)^{2 h_{1}}\left(\frac{\pi T_{R}}{\sinh \pi T_{R} x^{+}}\right)^{2 \bar{h}_{1}} .
$$

Fourier transforming this expression gives the propagator in momentum space. We compute the retarded/advanced propagators, which reduce to the Euclidean Matsubara propagator when restricted to the discrete Matsubara frequencies $\omega= \pm 2 \pi T k$, where $k \in \mathbb{Z}[12]$. To avoid singularities on the real axis the $\pm i \epsilon$ regularization prescription is implemented. This process leads to the advanced or retarded propagators depending on the sign of $i \epsilon$. 
For each sector we obtain $[11,13]$

$$
\begin{aligned}
G_{\Delta}^{ \pm}(\omega) & =\int_{-\infty}^{\infty} d x e^{-i \omega x}\left(\frac{\pi T}{\sinh (\pi T x \pm i \epsilon)}\right)^{2 \Delta} \\
& =(-1)^{\mp \Delta} \frac{(2 \pi T)^{2 \Delta-1}}{\Gamma(2 \Delta)} e^{\mp \omega / 2 T} \Gamma\left(\Delta+\frac{i \omega}{2 \pi T}\right) \Gamma\left(\Delta-\frac{i \omega}{2 \pi T}\right) .
\end{aligned}
$$

One can obtain eq. (0.5) by means of the following Mellin-Barnes type integral [14]

$$
\frac{1}{2 \pi i} \int_{-i \infty}^{+i \infty} e^{ \pm i \pi s} \xi^{s} \Gamma(\Delta+s) \Gamma(\Delta-s) d s=\Gamma(2 \Delta) e^{ \pm i \pi \Delta} \xi^{\Delta}(1-\xi \pm i \epsilon)^{-2 \Delta},
$$

where the integrand is defined on the principal branch of the complex logarithm.

After setting $\xi=e^{-2 \pi T x}, s=-i \omega / 2 \pi T$, and doing some manipulations, one obtains

$$
\begin{aligned}
\frac{1}{2 \pi} \int_{-\infty}^{\infty} d \omega e^{i \omega x}(2 \pi T)^{2 \Delta-1} \frac{1}{\Gamma(2 \Delta)} e^{ \pm \omega / 2 T} \Gamma\left(\Delta-\frac{i \omega}{2 \pi T}\right) \Gamma\left(\Delta+\frac{i \omega}{2 \pi T}\right) \\
=e^{ \pm i \pi \Delta}\left(\frac{\pi T}{\sinh (\pi T x \pm i \epsilon)}\right)^{2 \Delta}
\end{aligned}
$$

which is the inverse of the Fourier transform of eq. (0.5).

Next we calculate the momentum space finite temperature three-point function. Conformal symmetry fixes the form of the three-point function up to an overall constant. At finite temperature, the holomorphic part is

$$
\left\langle\phi_{h_{1}}\left(x_{1}^{+}\right) \phi_{h_{2}}\left(x_{2}^{+}\right) \phi_{h_{3}}(0)\right\rangle=\frac{(\pi T)^{l+m+n}}{\sinh ^{m}\left(\pi T x_{1}^{+}\right) \sinh ^{l}\left(\pi T x_{2}^{+}\right) \sinh ^{n}\left(\pi T x_{12}^{+}\right)} .
$$

Here $m=h_{3}+h_{1}-h_{2}, \quad l=h_{2}+h_{3}-h_{1}, \quad n=h_{1}+h_{2}-h_{3}$.

The holomorphic part of the momentum space three-point function follows by the Fourier transformation

$$
G_{m, n, l}\left(\omega_{1}, \omega_{2}\right)=\int_{-\infty}^{\infty} \int_{-\infty}^{\infty} d x_{1} d x_{2} \frac{(\pi T)^{l+m+n} e^{-i \omega_{1} x_{1}-i \omega_{2} x_{2}}}{\sinh ^{m}\left(\pi T x_{1}\right) \sinh ^{l}\left(\pi T x_{2}\right) \sinh ^{n}\left(\pi T x_{12}\right)} .
$$

Formally, the integral in eq. (0.9) is divergent so we work using the $i \epsilon$ prescription,

$$
G_{m, n, l}\left(\omega_{1}, \omega_{2}\right)=\int_{-\infty}^{\infty} \int_{-\infty}^{\infty} d x_{1} d x_{2} \frac{(\pi T)^{l+m+n} e^{-i \omega_{1} x_{1}-i \omega_{2} x_{2}}}{\sinh ^{m}\left(\pi T x_{1}+i \epsilon\right) \sinh ^{l}\left(\pi T x_{2}+i \epsilon\right) \sinh ^{n}\left(\pi T x_{12}-i \epsilon\right)}
$$

The signs of the $i \epsilon$ terms are chosen such that the integral is convergent.

By introducing the variable $u=x_{12}$, we can disentangle the arguments of the hyperbolic sines in the integrand as

$$
\begin{aligned}
G_{m, n, l}\left(\omega_{1}, \omega_{2}\right)=\int_{-\infty}^{\infty} \int_{-\infty}^{\infty} \int_{-\infty}^{\infty} & d u d x_{1} d x_{2} \delta\left(u-x_{1}+x_{2}\right) \\
& \times \frac{(\pi T)^{l+m+n} e^{-i \omega_{1} x_{1}-i \omega_{2} x_{2}}}{\sinh ^{m}\left(\pi T x_{1}+i \epsilon\right) \sinh ^{l}\left(\pi T x_{2}+i \epsilon\right) \sinh ^{n}(\pi T u-i \epsilon)} .
\end{aligned}
$$


Using the resolution of the identity,

$$
\delta(x)=\frac{1}{2 \pi} \int_{-\infty}^{\infty} d \omega e^{-i \omega x},
$$

one can rewrite eq. (0.11) as an integral of a product of Fourier-transformed two-point correlators

$$
G_{m, n, l}\left(\omega_{1}, \omega_{2}\right)=\int_{-\infty}^{\infty} \frac{d \omega}{2 \pi} G_{m / 2}^{+}\left(\omega_{1}-\omega\right) G_{n / 2}^{-}(\omega) G_{l / 2}^{+}\left(\omega_{2}+\omega\right)
$$

where, $G_{\Delta}^{ \pm}(\omega)$ is the Fourier transform of the two-point correlator with conformal weight $\Delta$ as given in eq. (0.5). It is easy to see that the above expression agrees with the one for the extremal three-point correlation function computed in [15], when either $m=0, n=0$, or $l=0$. It also reduces to the advanced or retarded propagator by setting one of the conformal weights to zero and the remaining two equal to each other. For example, if one chooses $h_{3}=0$ and $h_{1}=h_{2}$ then

$$
G_{0,2 h_{1}, 0}\left(\omega_{1}, \omega_{2}\right)=\delta\left(\omega_{1}+\omega_{2}\right) G_{h_{1}}^{-}\left(\omega_{1}\right) .
$$

We proceed by substituting the expression for $G_{\Delta}^{ \pm}$into eq. (0.13),

$$
\begin{aligned}
G_{m, n, l}\left(\omega_{1}, \omega_{2}\right)=(-1)^{(-m+n-l) / 2} & \frac{(2 \pi T)^{m+n+l-3}}{\Gamma(m) \Gamma(n) \Gamma(l)} e^{\left(\omega_{1}+\omega_{2}\right) / 2 T} \\
\times \int_{-\infty}^{\infty} \frac{d \omega}{2 \pi} & \left(e^{-\omega / 2 T} \Gamma\left(\frac{m}{2}+i \frac{\omega_{1}}{2 \pi T}-i \frac{\omega}{2 \pi T}\right)\right. \\
\times & \Gamma\left(\frac{m}{2}-i \frac{\omega_{1}}{2 \pi T}+i \frac{\omega}{2 \pi T}\right) \Gamma\left(\frac{n}{2}+i \frac{\omega}{2 \pi T}\right) \Gamma\left(\frac{n}{2}-i \frac{\omega}{2 \pi T}\right) \\
\times & \left.\Gamma\left(\frac{l}{2}+i \frac{\omega_{2}}{2 \pi T}+i \frac{\omega}{2 \pi T}\right) \Gamma\left(\frac{l}{2}-i \frac{\omega_{2}}{2 \pi T}-i \frac{\omega}{2 \pi T}\right)\right) .
\end{aligned}
$$

With the simple change of variables $\omega=i 2 \pi T s$ we recognize the above integral as the defining integral of the Meijer-G function on the first path $[16,17]$

$$
\begin{aligned}
G_{m, n, l}\left(\omega_{1}, \omega_{2}\right)=(-1)^{(m-n+l) / 2} & \frac{(2 \pi T)^{m+n+l-2}}{\Gamma(m) \Gamma(n) \Gamma(l)} e^{-\left(\omega_{1}+\omega_{2}\right) / 2 T} \\
& \times G_{3,3}^{3,3}\left(\begin{array}{c}
1-\frac{m}{2}-i \frac{\omega_{1}}{2 \pi T}, 1-\frac{n}{2}, 1-\frac{l}{2}+i \frac{\omega_{2}}{2 \pi T} \\
\frac{m}{2}-i \frac{\omega_{1}}{2 \pi T}, \frac{n}{2}, \frac{l}{2}+i \frac{\omega_{2}}{2 \pi T}
\end{array}\right) .
\end{aligned}
$$

One easily checks that the convergence conditions for this function are satisfied because $p+q<2(r+s),|\arg z|<(r+s-(p+q) / 2) \pi$ for $G_{r, s}^{p, q}$ with argument $z$, and the poles of the Gamma functions are on the right locations [17].

For general values of $m, n$ and $l$ this function has to be evaluated numerically. For integer values of these parameters the function can be evaluated analytically. For example, 
with $m=1, n=3$ and $l=2$ we get from eq. (0.16)

$$
\begin{aligned}
& G_{1,3,2}\left(\omega_{1}, \omega_{2}\right) \\
& =\frac{\pi^{2}}{6\left(e^{-\frac{\omega_{2}}{T}}+1\right)\left(1-e^{-\frac{\omega_{1}}{T}}\right)}\left(\pi^{2} T^{2}+\omega_{2}^{2}\right)\left(9 \pi^{2} T^{2}+\omega_{2}^{2}\right) \\
& \quad+\frac{\pi^{2}}{6\left(e^{\frac{-\omega_{1}-\omega_{2}}{T}}+1\right)\left(1-e^{\frac{\omega_{1}}{T}}\right)}\left(\pi^{2} T^{2}+\left(\omega_{1}+\omega_{2}\right)^{2}\right)\left(9 \pi^{2} T^{2}+3 \omega_{1}^{2}+\omega_{2}^{2}-2 \omega_{1} \omega_{2}\right) .
\end{aligned}
$$

We see that the finite-temperature three-point function has a similar frequency dependence as the greybody factors found in the absorption cross-section in [18]. It is also easy to check that the zero temperature limit of eq. (0.17) precisely gives the Fourier transform of the correlation function on the plane,

$$
\lim _{T \rightarrow 0} G_{1,3,2}\left(\omega_{1}, \omega_{2}\right)=\int_{-\infty}^{\infty} d x_{1} d x_{2} e^{-i \omega_{1} x_{1}-i \omega_{2} x_{2}} \frac{1}{\left(x_{1}+i \epsilon\right)^{1}} \frac{1}{\left(x_{2}+i \epsilon\right)^{2}} \frac{1}{\left(x_{1}-x_{2}-i \epsilon\right)^{3}} .
$$

The result in eq. (0.16) holds for any (even strongly coupled) field theory with a 2D CFT holographic dual. It would be interesting to see if this result agrees with the bulk calculation of three-point functions in the near-NHEK geometry, as proposed by the Kerr/CFT correspondence [6, 7], beyond the extremal case considered in [15]. Work in this direction is in progress.

\section{Acknowledgments}

We thank Katrin Becker, Tom Hartman, and Andrew Strominger for useful discussions. This work was supported by the NSF under the grants PHY-0505757, DMS-1159404 and DGE-1252521 and by Texas A\&M University.

Open Access. This article is distributed under the terms of the Creative Commons Attribution License (CC-BY 4.0), which permits any use, distribution and reproduction in any medium, provided the original author(s) and source are credited.

\section{References}

[1] O. Aharony, S.S. Gubser, J.M. Maldacena, H. Ooguri and Y. Oz, Large-N field theories, string theory and gravity, Phys. Rept. 323 (2000) 183 [hep-th/9905111] [INSPIRE].

[2] N. Iqbal, H. Liu and M. Mezei, Lectures on holographic non-Fermi liquids and quantum phase transitions, arXiv:1110.3814 [INSPIRE].

[3] S. Sachdev, What can gauge-gravity duality teach us about condensed matter physics?, Ann. Rev. Condensed Matter Phys. 3 (2012) 9 [arXiv:1108.1197] [InSPIRE].

[4] C.P. Herzog, Lectures on holographic superfluidity and superconductivity, J. Phys. A 42 (2009) 343001 [arXiv:0904.1975] [INSPIRE]. 
[5] G.T. Horowitz, Introduction to holographic superconductors, Lect. Notes Phys. 828 (2011) 313 [arXiv:1002.1722].

[6] M. Guica, T. Hartman, W. Song and A. Strominger, The Kerr/CFT correspondence, Phys. Rev. D 80 (2009) 124008 [arXiv:0809.4266] [InSPIRE].

[7] I. Bredberg, C. Keeler, V. Lysov and A. Strominger, Cargese lectures on the Kerr/CFT correspondence, Nucl. Phys. Proc. Suppl. 216 (2011) 194 [arXiv:1103.2355] [InSPIRE].

[8] G. Compere, The Kerr/CFT correspondence and its extensions: a comprehensive review, Living Rev. Rel. 15 (2012) 11 [arXiv:1203.3561] [INSPIRE].

[9] J. Casalderrey-Solana, H. Liu, D. Mateos, K. Rajagopal and U.A. Wiedemann, Gauge/string duality, hot QCD and heavy ion collisions, arXiv:1101.0618 [INSPIRE].

[10] J.M. Maldacena and A. Strominger, Universal low-energy dynamics for rotating black holes, Phys. Rev. D 56 (1997) 4975 [hep-th/9702015] [INSPIRE].

[11] S.S. Gubser, Absorption of photons and fermions by black holes in four-dimensions, Phys. Rev. D 56 (1997) 7854 [hep-th/9706100] [INSPIRE].

[12] D.T. Son and A.O. Starinets, Minkowski space correlators in AdS/CFT correspondence: recipe and applications, JHEP 09 (2002) 042 [hep-th/0205051] [INSPIRE].

[13] I. Bredberg, T. Hartman, W. Song and A. Strominger, Black hole superradiance from Kerr/CFT, JHEP 04 (2010) 019 [arXiv:0907.3477] [INSPIRE].

[14] B. Jantzen, New proofs for the two Barnes lemmas and an additional lemma, J. Math. Phys. 54 (2013) 012304 [arXiv:1211.2637] [INSPIRE].

[15] M.P. van Daalen, J. Schaye, C.M. Booth and C.D. Vecchia, The effects of galaxy formation on the matter power spectrum: a challenge for precision cosmology, Mon. Not. Roy. Astron. Soc. 415 (2011) 3649 [arXiv:1104.1174] [INSPIRE].

[16] J. Erdelyi, W. Magnus, F. Oberhettinger and F.G. Tricomi, Higher transcendental functions, volume I, McGraw-Hill Book Company Inc., U.S.A. (1953).

[17] R.I.M. Gradshteyn, Table of integrals, series, and products, Academic Press Inc., U.S.A. (1994).

[18] J.M. Maldacena and A. Strominger, Black hole grey body factors and D-brane spectroscopy, Phys. Rev. D 55 (1997) 861 [hep-th/9609026] [INSPIRE]. 